\title{
Fabrication of polypropylene/lignin blend sponges via thermally induced phase separation for the removal of oil from contaminated water
}

\author{
Abeer Alassod ${ }^{1,2}$. Syed Rashedul Islam ${ }^{1} \cdot$ Amjad Farooq $^{1} \cdot$ Guangbiao Xu $^{1}$
}

Received: 3 July 2020 / Accepted: 19 August 2020 / Published online: 27 August 2020

(c) Springer Nature Switzerland AG 2020

\begin{abstract}
Polypropylene is widely used in oil spillage cleanup due to excellent characteristics. However, polypropylene is not renewable and biodegradable, which is unacceptable to introduce new pollutants while solving environmental disasters. Therefore, there is a high demand to explore a low-cost, environmentally friendly, and renewable technique for the fabrication of porous materials. In this work, lignin was chosen as the second blend in the polymer matrix. Polypropylene and renewable lignin sponges were successfully prepared using simple, inexpensive, controllable, scalable, and an environment-friendly method named thermally induced phase separation (TIPS). The surface morphology of obtained sponges was investigated using FTIR and SEM. FTIR analysis indicated that PP and lignin were physically blended. SEM analysis observed an interconnected porous network that acts as a capture site of oil, and lignin merged into PP. The contact angle of PP, PP5L, PP10L, PP15L, and PP20L was found to be $127.4^{\circ}, 118.71^{\circ}, 113.89^{\circ}, 109.45^{\circ}$, and $107^{\circ}$, respectively. Furthermore, polypropylene/lignin sponges have good adsorption ability toward oils compared to polypropylene itself. The research detected that the highest oil sorption tests exhibited by PP10L sponge, which could absorb $983 \%$ of soybean oil, $788 \%$ of engine oil, and $550 \%$ of lubricating oil in the oil system, with high oil retention more than $90 \%$ after 24-h dripping. Besides, the results revealed that temperature has a significant effect on oil absorption. All of these features make polypropylene/lignin blend sponges promising sorbents for the oil spills cleanup, not only for oil recovery but also helps in cleaning the environment.
\end{abstract}

Keywords Polypropylene - Lignin · Oil sorption - Thermally induced phase separation · Thermal stability

\section{Introduction}

Oil is a part of the natural environment for many decades and is a naturally occurring substance with the rapid development of oil production, transportation, exploration, and storage [1-4]. The risk of oil spillage increased with the potential to create a notable environmental problem and also a great waste of energy [5-8]. Therefore, many methods have adopted to clean and recycle spilled oil. Among these methods, mechanical extraction by sorption materials is considered one of the most popular methods $[9,10]$. An ideal oil sorbent material should have hydrophobicity, oleophilicity, sorption capacity, buoyancy, good oil-water selectivity, and reusability [11, 12]. At present, synthetic polymer materials, such as PP, have been widely used as oil sorbents due to unique features such as excellent mechanical and chemical properties as well as the ease of processing; density is less than that of water [13]. Further, PP has hydrophobic and oleophilic features, which make it potentially promising for materials for oil

$\triangle$ Guangbiao Xu, Guangbiao_xu@dhu.edu.cn | ${ }^{1}$ Key Laboratory of Textile Science and Technology of Ministry of Education, College of Textiles, Donghua University, Shanghai 201620, China. ${ }^{2}$ Department of Textile Engineering, Mechanical and Electrical Engineering University, Damascus, Syria. 
spill cleanup [14-16]. Nevertheless, these materials have some limitations that degrade very slowly, and that is considered to be mainly responsible for the phenomenon known as white pollution, which results in the accumulation of plastics on earth [17].

Moreover, the current price of PP has been estimated to be around \$1210/ton [18]. Therefore, partial replacement of PP with low-cost, environmentally friendly, and renewable materials was the demand to use renewable sources for bio-economic perspective consideration. Recently, great attention to the development of renewable resources based on natural biomass sources has been an ever-growing field over the last decade [19]. Biocomposite is derived from biomass such as lignin, starch, and natural cellulose, which has been the subject of many studies due to the advantages such as economic and environmental factors [20,21]. Among natural abundant and biomass materials, lignin is the second most abundant natural, simple biopolymer on earth after cellulose, which is still considered a by-product and burns as a low-value-added fuel $[22,23]$. Currently, around 50 million tons of lignin is produced annually as a by-product of the pulp-and-paper industry. Only a small fraction (about $2 \%$ ) is effectively utilized, and most of the lignin is burned or discharged into rivers, thereby causing serious environmental pollution and grievous waste of renewable resources [24, 25]. Lignin gained considerable attention for various applications. Chemically, lignin is a complex, three-dimensional amorphous polymeric material with aliphatic and aromatic subunits. Aromatic groups impart a polar character to polymers and contribute to their incompatibility with nonpolar polymers such as PP [26], polyethylene (PE) [27, 28], and polyester (PS) [29].

Many authors investigated the field of PP/lignin systems in the past [17, 30-32]. The treatment of oily wastewater is still a big task and also indispensable to developed efficient large-scale and economical methods to fabricate sorbents from low-cost materials. Hence, to solve the problems of high-cost devices and long production time, phase separation of the polymer solution is one of the useful methods to prepare porous polymers with interconnected porous structure. Several methods to induce phase separation are adopted; thermally induced phase separation method (TIPS) is one among those methods [33-36]. TIPS method is considered as a simple and clean process that also surpasses the extra effort needed for the removal of the template molecules. Another attractive feature of the TIPS method over other conventional techniques is the formation of an intrinsically interconnected porous network, that is controllable, versatile, convenient, and scalable [37].

Herein, this study aims to develop low-cost and environmentally friendly sponges prepared using lignin and PP via using thermally induced phase separation (TIPS). To
Table 1 Properties of the test oils

\begin{tabular}{lccc}
\hline Oil & Soybean oil & $\begin{array}{l}\text { Engine oil } \\
(15 \mathrm{~W}-40)\end{array}$ & Lubricating oil \\
\hline Viscosity $(\mathrm{mPa} \mathrm{s})$ & 65.3 & 234.5 & 21.70 \\
Density $\left(\mathrm{g} \mathrm{cm}^{-3}\right)$ & 0.92 & 0.87 & 0.82 \\
Surface tension $\left(\mathrm{mN} \mathrm{m}^{-1}\right)$ & 33.41 & 30.78 & 28.11 \\
\hline
\end{tabular}

Table 2 Produced composites and their sample codes

\begin{tabular}{lcc}
\hline Sample name & PP \% & Lignin \% \\
\hline PP & 100 & 0 \\
PP5L & 95 & 5 \\
PP10L & 90 & 10 \\
PP15L & 85 & 15 \\
PP20 L & 80 & 20 \\
\hline
\end{tabular}

best of the knowledge, using lignin incorporated in PP via thermally induced phase separation (TIPS) has not been reported yet. The outcomes from this research are believed to provide an essential reference in the practical application of polypropylene and lignin blend sponges in oil spill management.

\section{Materials and methods}

\subsection{Materials}

PP pellets (PP), lignin (L), acetone, decalin, and 1-butanol were purchased from Aladdin, Shanghai, PR China. Three different oils, namely engine oil, soybean oil, and lubricating oil, were used. The densities and surface tensions of those oils were examined using dynamic contact angle tester (DCAT1), and ASNB2 Digital Rotary Viscometer tested the viscosities. Every test was repeated three times to obtain an average value. The test temperature was maintained at $22 \pm 2{ }^{\circ} \mathrm{C}$. The properties of the studied oils are reported in Table 1 .

\subsection{Fabrication of PP/lignin blend sponges}

The fabrication of polypropylene/lignin blend sponges by the thermally induced phase separation technique (TIPS) was accomplished as follows. Polypropylene pellet was dissolved in the mixed solvent of decalin and 1-butanol at $115^{\circ} \mathrm{C}$, and lignin was added manually in solution. Then, the solution was cooled at $20^{\circ} \mathrm{C}$, resulting in the phase separation of the polymer in the form of a sponge. The sponge was immersed with acetone and subsequently dried under vacuum. The addition levels of dried lignin 
were set at $0,5,10,15$, and $20 \mathrm{wt} \%$ of the total weight of polypropylene pellets, as reported in Table 2 . The general protocol for the procedure for the fabrication of sponge is illustrated in Fig. 1.

\subsection{Characterization}

\subsubsection{Morphology}

A scanning electron microscope of polypropylene/lignin blend sponges' surface was characterized by using scanning electron microscopy (SEM) (FLEX SEM1000, Hitachi, Japan). For SEM observation, samples were cut, then fixed on double tape, and after that plated with a thin film of gold before measurement.

\subsubsection{Fourier transform infrared measurement}

The infrared spectrum was performed using the FTIR spectrometer (Nicolet 6700, Thermo Fisher, USA). The FTIR spectra were recorded in the range $400-4000 \mathrm{~cm}^{-1}$ wavenumber.

\subsubsection{The oil absorption experiment}

The absorption ability was studied by weighing the samples before $\left(W_{i}\right)$ and after $\left(W_{t}\right)$ immersing them in oils (soybean, engine) as percent weight gain $(Q)[38,39]$. Samples were lifted and placed with free oil dripping out for $24 \mathrm{~h}$, weight was measured $\left(W_{d}\right)$, then the retention was calculated $(R)$ [40], and the weight gain and retention were calculated according to Eqs. (1) and (2):

$Q=\frac{W_{t}-W_{i}}{W_{i}} \times 100$
$R=\frac{W_{d-W_{i}}}{W_{t}-W_{i}} \times 100$

The ability to reuse the sorbents for oil sorption was studied using soybean oil, the sorbents with oil were squeezed, and then squeezed sorbent was again used with the same procedure as described above. The sorptionsqueezing process was repeated many times under identical conditions to evaluate the reusability of the sorbent.

\section{Results and discussion}

\subsection{Morphology}

In all tested samples, the three-dimensional interconnected porous structures were investigated by SEM, as depicted in Fig. 2. This is explained to the phase separation of the polymer solution during the cooling process, in which polymer-rich regions contributed to the formation of skeletons. The porous structures provided sufficiently storage space to capture the oil. The pore volume decreased by squeezing the sorbent, causing the recovery of the absorbed oil. The figure revealed the characterization of blend sponge that lignin achieved incorporation in the PP blend successfully [41]. Moreover, the introduction of lignin with three-dimensional structures effectively supported the micro-/nanoscale structures [42]. It is clearly obvious that pure polypropylene had a smooth surface, as shown in Fig. 2a, while with addition, lignin assembles a rough surface, as shown in Fig. $2 b-e$, which indicates the small lignin particles embedded in polypropylene matrix successfully [41].

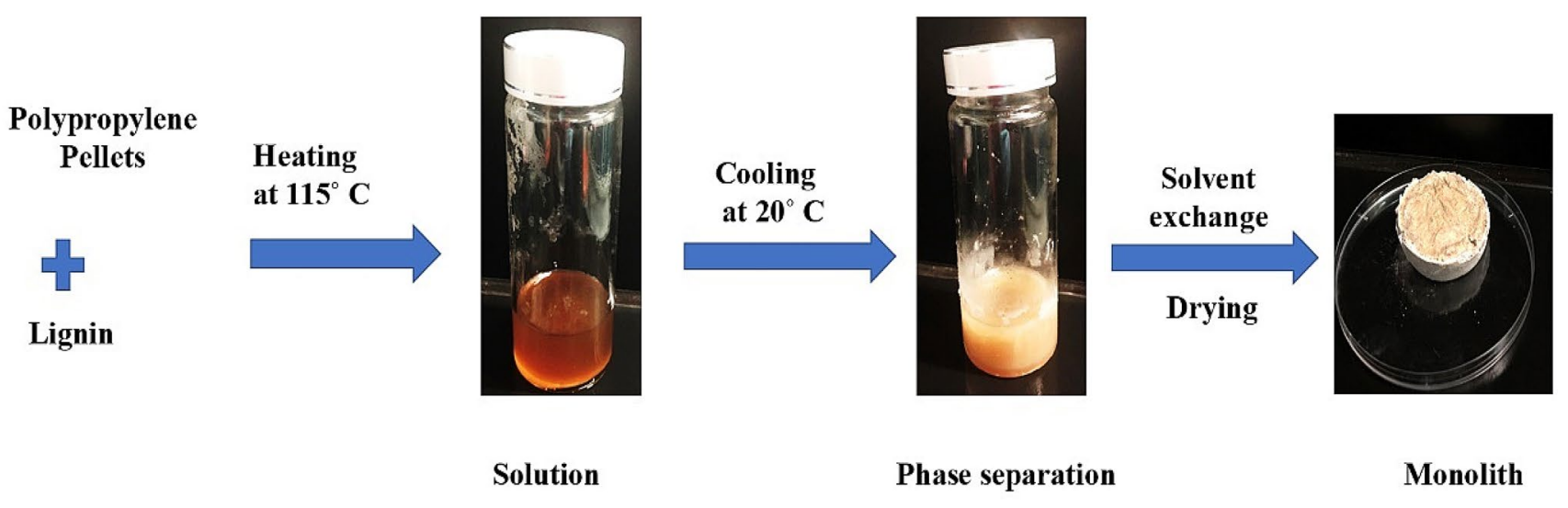

Fig. 1 A general protocol for the preparation of polypropylene/lignin blend sponges by a thermally induced phase separation technique 

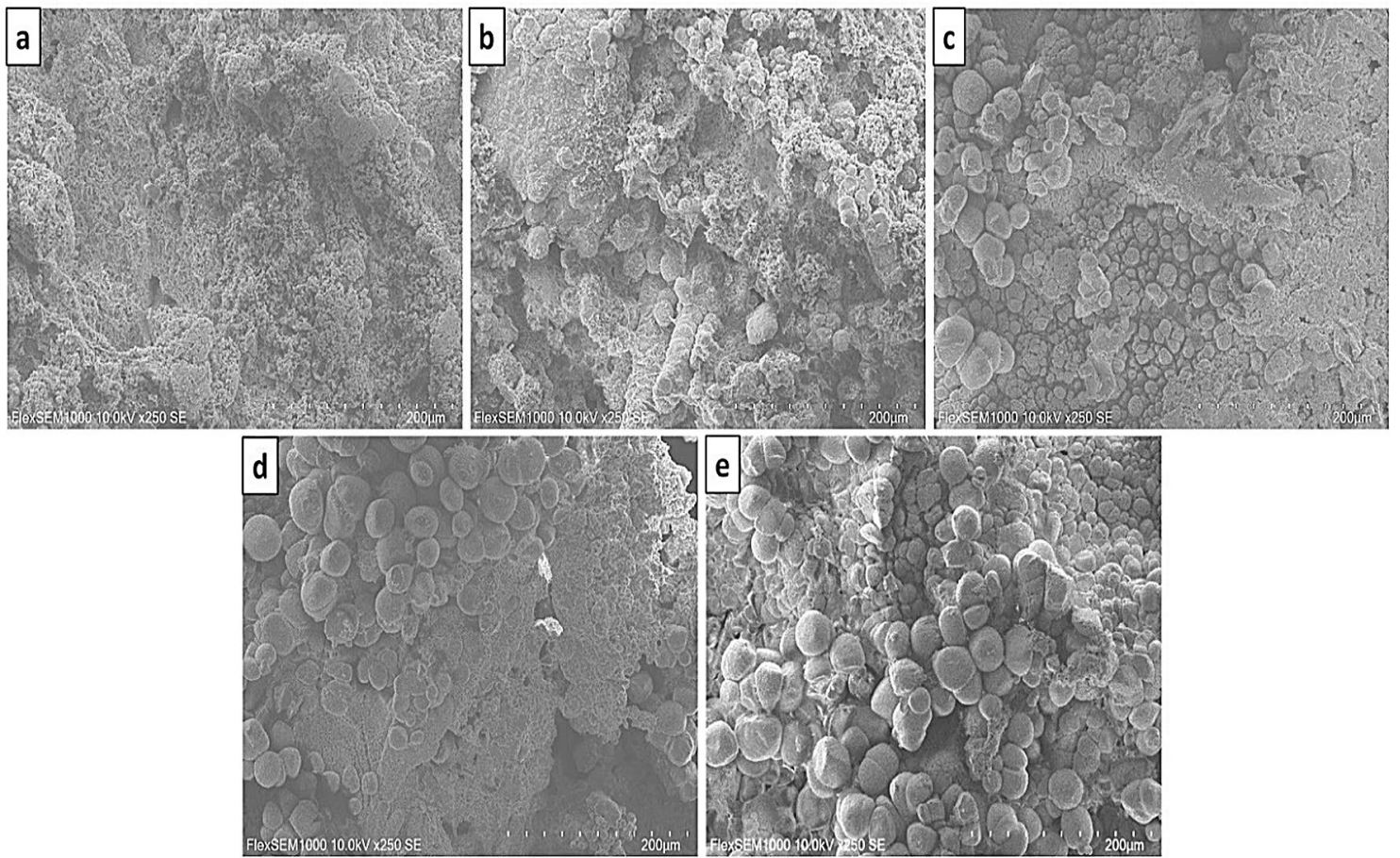

Fig. 2 SEM image a PP, b PP5L, c PP10L, d PP15L, e PP20L

a

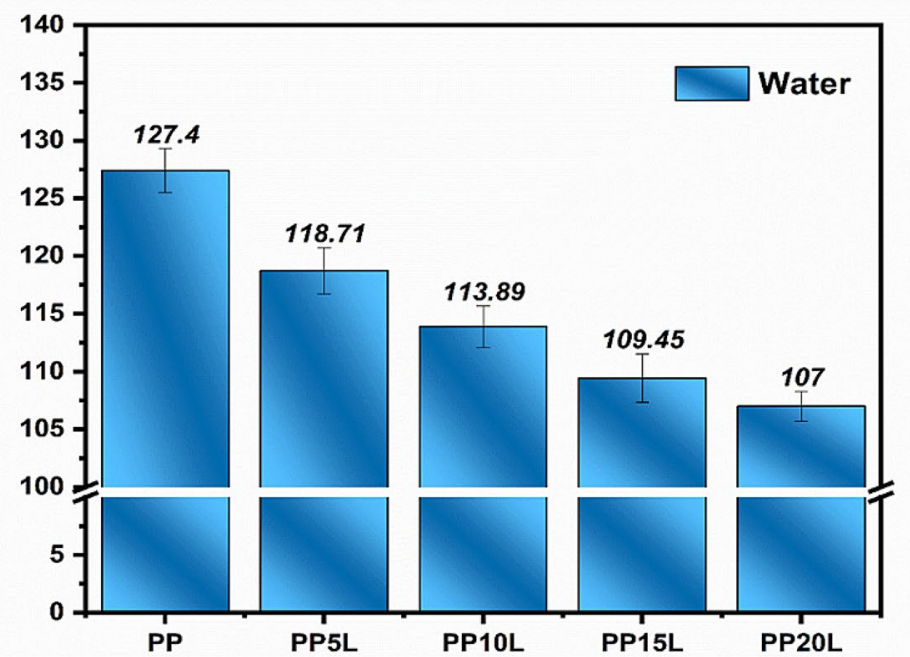

b

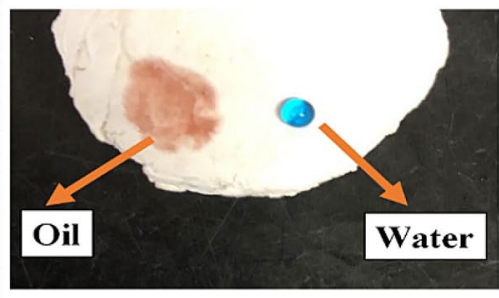

Fig. 3 a Water contact angle of polypropylene and polypropylene/lignin blend sponges, b Surface wettability of blend sorbent. Engine oil (dyed in red) and water (dyed in blue with methylene blue) were spotted on the surface of the sorbent

\subsection{Hydrophobicity and lipophilic}

The wettability "contact angle result" of polypropylene and polypropylene/lignin was performed to study the behavior of different lignin content in the polypropylene matrix, as displayed in Fig. 3a. PP showed a hydrophobic feature with a contact angle around $127.40^{\circ}$, while the addition of 5 wt $\%$ of lignin in polymer matrix reduced contact angle for value around $118.71^{\circ}$. The obtained results may be attributed to the contribution of the several free polar groups from lignin [43]. With increasing lignin, loading in the PP matrix to values above $10 \mathrm{wt} \%$ provides contact angles for PP10L, PP15L, and PP20L, which were $113.89^{\circ}, 109.45^{\circ}$, and $107^{\circ}$, respectively. However, the contact angle for these sorbents is more than $90^{\circ}$ according to the standard definition of hydrophobic materials [44]. These sorbents also

\section{SN Applied Sciences}


have hydrophobic features. On the another side to indicate lipophilic feature for sorbents, it was obviously noticed that tested sorbents immediately absorbed oil droplets, as shown in Fig. $3 b$.

\subsection{Fourier transform infrared spectroscopy analysis}

Fourier transform infrared (FTIR) measurement of polypropylene, lignin, and their composites polypropylene/ lignin with different proportions between wavenumbers 4000 and $500 \mathrm{~cm}^{-1}$ is shown in Fig. 4 . FTIR spectra of lignin showed the wide band in the range between 3400 and $3500 \mathrm{~cm}^{-1}$ due to the hydroxyl groups (O-H stretching) [45], following a range of bands around $2936 \mathrm{~cm}^{-1}$ (C-H stretching) and $2846 \mathrm{~cm}^{-1}$ ( $\mathrm{C}-\mathrm{H}$ tensor). One of the essential peaks in all polypropylene/lignin is the band at a range between 3400 and $3500 \mathrm{~cm}^{-1}$, which becomes clearly observed when increasing the addition of lignin in polypropylene matrix (PP20L > PP15L > PP10L > PP5L). Furthermore, from this figure it can be seen that PP had peaks at $2950 \mathrm{~cm}^{-1}, 2916 \mathrm{~cm}^{-1}, 2866 \mathrm{~cm}^{-1}$, and $2836 \mathrm{~cm}^{-1}$, which represented (C-H stretching); also two bands are observed at $1373 \mathrm{~cm}^{-1}$ (syringyl group) and $1360 \mathrm{~cm}^{-1}$ $\left(\mathrm{C}-\mathrm{H}_{3}\right.$ bonding and $\mathrm{C}=\mathrm{O}$ stretch) in polypropylene/lignin structure like polypropylene [46].

It can be observed from a typical FTIR spectrum of added lignin in polypropylene that the composition of the bonds did change but increased the intensity of the peaks. Other important structures in lignin are aromatic rings because aromatic skeleton vibrations at peaks $1597 \mathrm{~cm}^{-1}$ and $1510 \mathrm{~cm}^{-1}$ are visible PP/lignin [47]. Also, the presence of new bands around $1710 \mathrm{~cm}^{-1}$ in the carbonyl region can be associated with conjugated carbonyl stretching [48]. Lignin was detected at $1250 \mathrm{~cm}^{-1}, 1210 \mathrm{~cm}^{-1}, 1115 \mathrm{~cm}^{-1}$, and $1037 \mathrm{~cm}^{-1}$ corresponding to syringyl absorptions, guaiacyl ring breathing, which was observed in the spectrum of polypropylene/lignin (Fig. 4).

\subsection{Oil sorption}

Polypropylene/lignin blend sponges were prepared via the TIPS method that are promising sorbents for oil cleanup because of their 3-D interconnected macroporous structure, hydrophobicity, super-oleophilicity, and also because incorporated lignin provides micro-/nanoscale structures, respectively, as discussed above [42]. In this case, it was observed through the experimental test that the adsorption process speed is slower, which indicates the presence of large holes and small macropores. That increase in absorbed oil into a tested sample is a vital key for the fast removal of spilled oils. The weight gain of tested samples in the oil system and oil/water system was measured, as shown in Fig. 5a. The weight gain of PP, PP5L, PP10L, PP15L, and PP20L for soybean oil is $480 \%, 685 \%, 983 \%$, $875 \%$, and $788 \%$, respectively. The engine oil weight gain of these sorbents indicates a similar tendency that is $388 \%$, $543 \%, 788 \%, 701 \%$, and $645 \%$, respectively. Besides, lubricating oil weight gain of these sorbents indicates a similar tendency that is $275 \%, 320 \%, 550 \%, 451 \%$, and $385 \%$, respectively. Figure $5 b, c$, and e shows the weight gain of sorbents in the oil-water system. It was observed that
Fig. 4 FTIR spectra of polypropylene, lignin, and polypropylene/lignin blend sponges

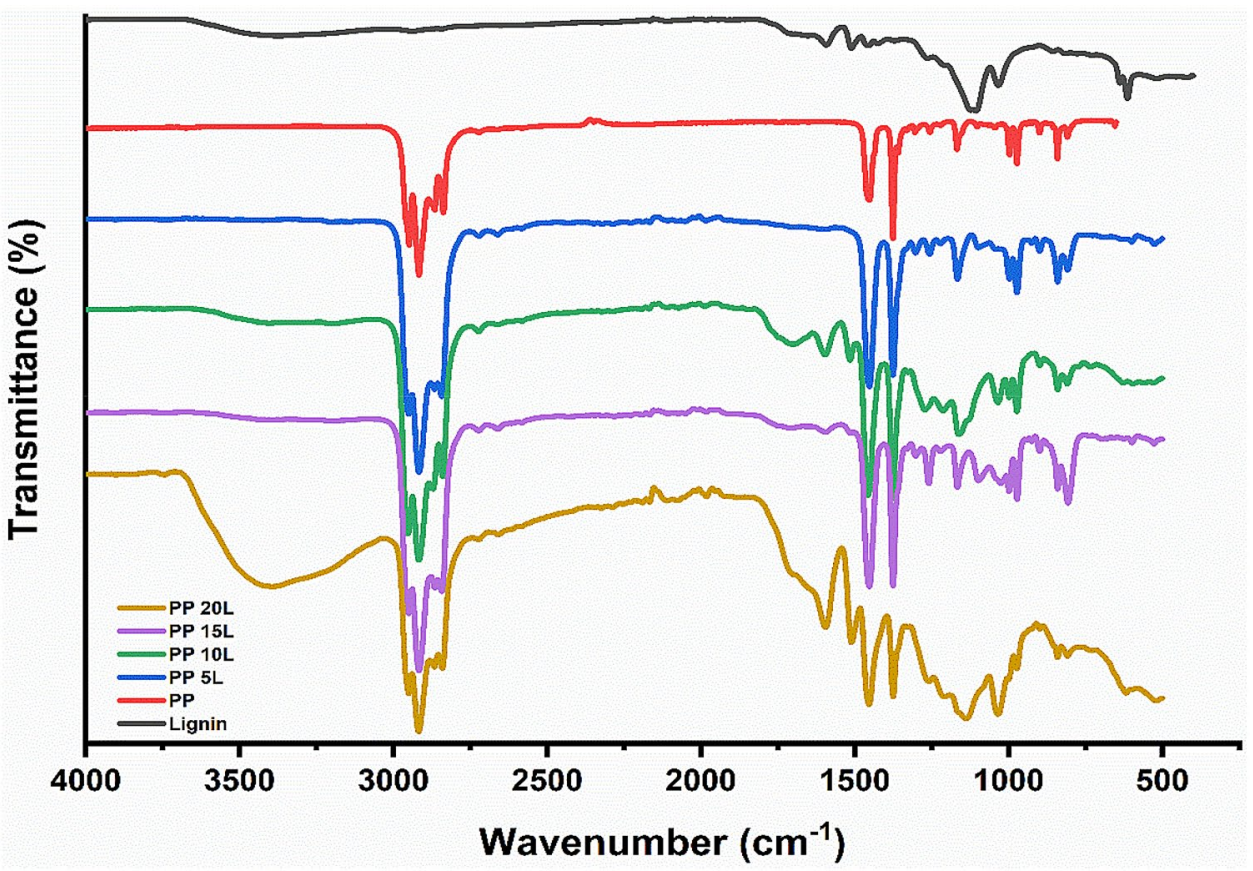



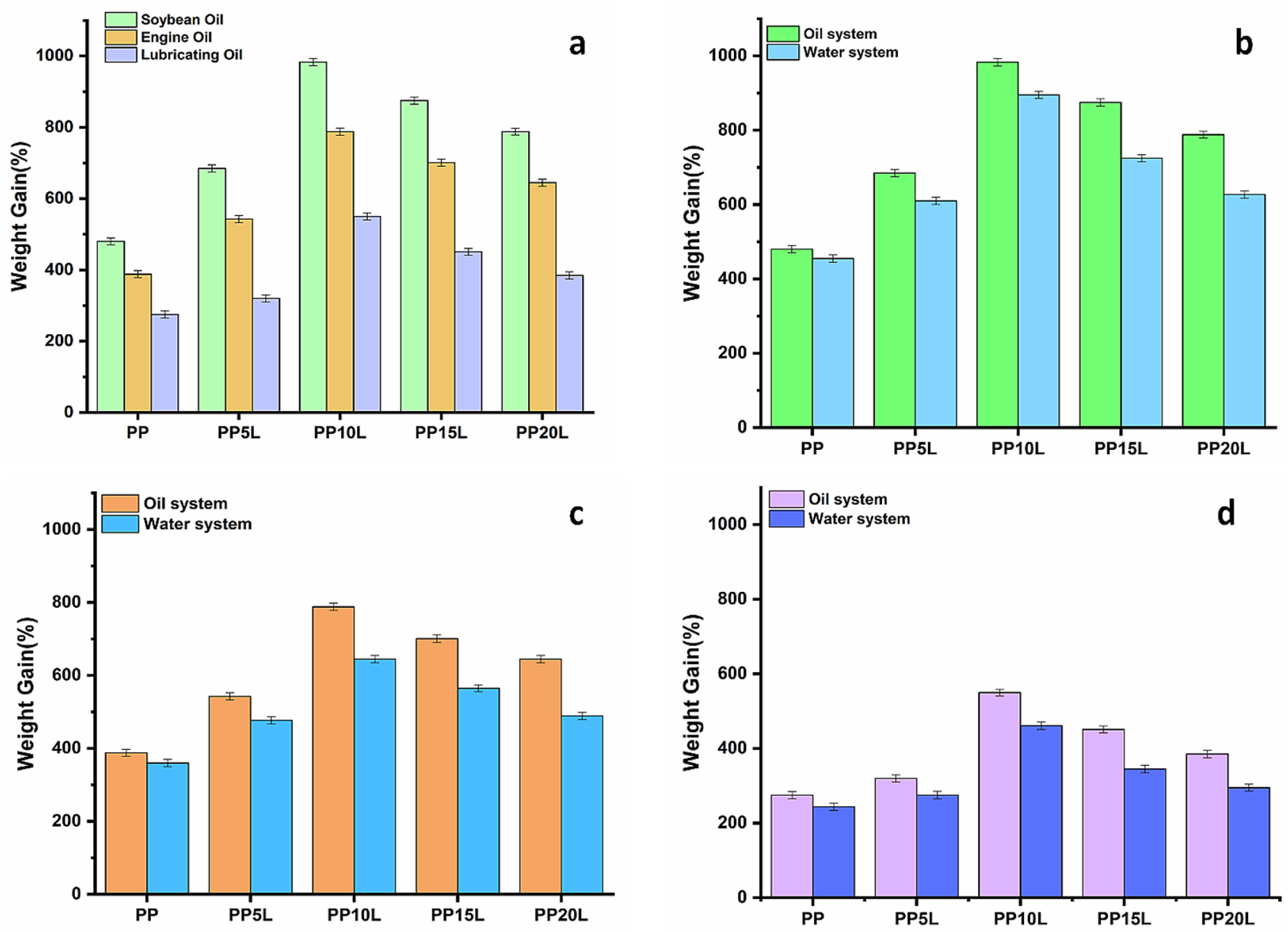

Fig. 5 Absorption capacities of polypropylene and polypropylene/lignin blend sponge a oil system, b soybean oil system/water system, c engine oil system/water system, d lubricating oil system/water system

weight gain for soybean oil is $455 \%, 610 \%, 895 \%, 725 \%$, and $627 \%$, respectively, for engine oil; the tendency is the same behavior, that is $360 \%, 477 \%, 645 \%, 565 \%$, and $489 \%$, respectively. And for lubricating oil, the tendency is the same behavior, that is $240 \%, 275 \%, 461 \%, 345 \%$, and $295 \%$, respectively. All obtained results showed higher oil sorption in the oil system when compared to the oil/water system. It was observed with the increased value of lignin more than $10 \mathrm{wt} \%$, and there was a slight reduction in oil sorption. This result corroborates the contact angle results. Even that still PP15L and PP20L showed an improvement in sorption capacity compared to the blank one for both systems, it was seen that there is an increase in oil sorption with increased addition of lignin content. All polymer modified by lignin had to show the positive impact of lignin on oil sorption in the system. Lignin slightly reduced the hydrophobic character of the polymer matrix due to the polar groups in the structure. Still, it also has aromatic groups obtained from benzene, which can favor the sorption of organic compounds [49].
High oil retention ability is an essential feature to keep oil encapsulated in the sorbent so that the sorbent can be relocated from the water to a nominated area without losing the oil into the surrounding so that it avoids the second contaminant. The oil retention after $24 \mathrm{~h}$ dripping for tested sorbents was measured as the values listed in Table 3. The oil retention for PP, PP5L, PP10L, PP15L, and PP20L for soybean oil was $78.57 \%, 88.04 \%, 92.20 \%$, $90.96 \%$, and $89.11 \%$, respectively. The engine oil retention

Table 3 Oil retention of PP and PP/lignin blend sponges

\begin{tabular}{lllr}
\hline Sample code & $\begin{array}{l}\text { Soybean oil } \\
\text { retention (\%) }\end{array}$ & $\begin{array}{l}\text { Engine oil } \\
\text { retention (\%) }\end{array}$ & $\begin{array}{l}\text { Lubricating oil } \\
\text { retention (\%) }\end{array}$ \\
\hline PP & $78.57(1.34)$ & $82.97(1.77)$ & $72.70(1.93)$ \\
PP5L & $88.04(1.47)$ & $88.92(1.89)$ & $75(1.87)$ \\
PP10L & $92.20(1.17)$ & $93.87(1.32)$ & $90.90(1.39)$ \\
PP15L & $90.96(1.34)$ & $91.61(1.92)$ & $83.30(1.77)$ \\
PP20L & $89.11(1.56)$ & $89.89(1.45)$ & $78.94(1.71)$ \\
\hline
\end{tabular}


of these sorbents indicates a similar tendency, that is $82.97 \%, 88.92 \%, 93.87 \%, 91.61 \%$, and $89.89 \%$, respectively. And lubricating oil retention of these sorbents indicates a similar tendency, that is $72.70 \%, 75 \%, 90.90 \%, 83.30 \%$, and $78.94 \%$, respectively. Generally speaking, it was noted that tested samples absorbed a higher amount of soybean oil than the engine oil and lubricating oil. This could be attributed to the fact that soybean oil is heavier than engine oil within the same unit volume. On the other hand, soybean oil was more ready to drip out from tested sorbents than engine oil. The draining takes place when the capillary pressure is insufficient to capture the weight of oils. The heavy nature of soybean oil combined with its lower viscosity than engine oil was favorable for the dipping process [50].

Figure 6a shows a mixture of oil and water, in which the colorless transparent part is water and the colored part is oil. As shown in Fig. $6 \mathrm{~b}$, the blend material floats on the
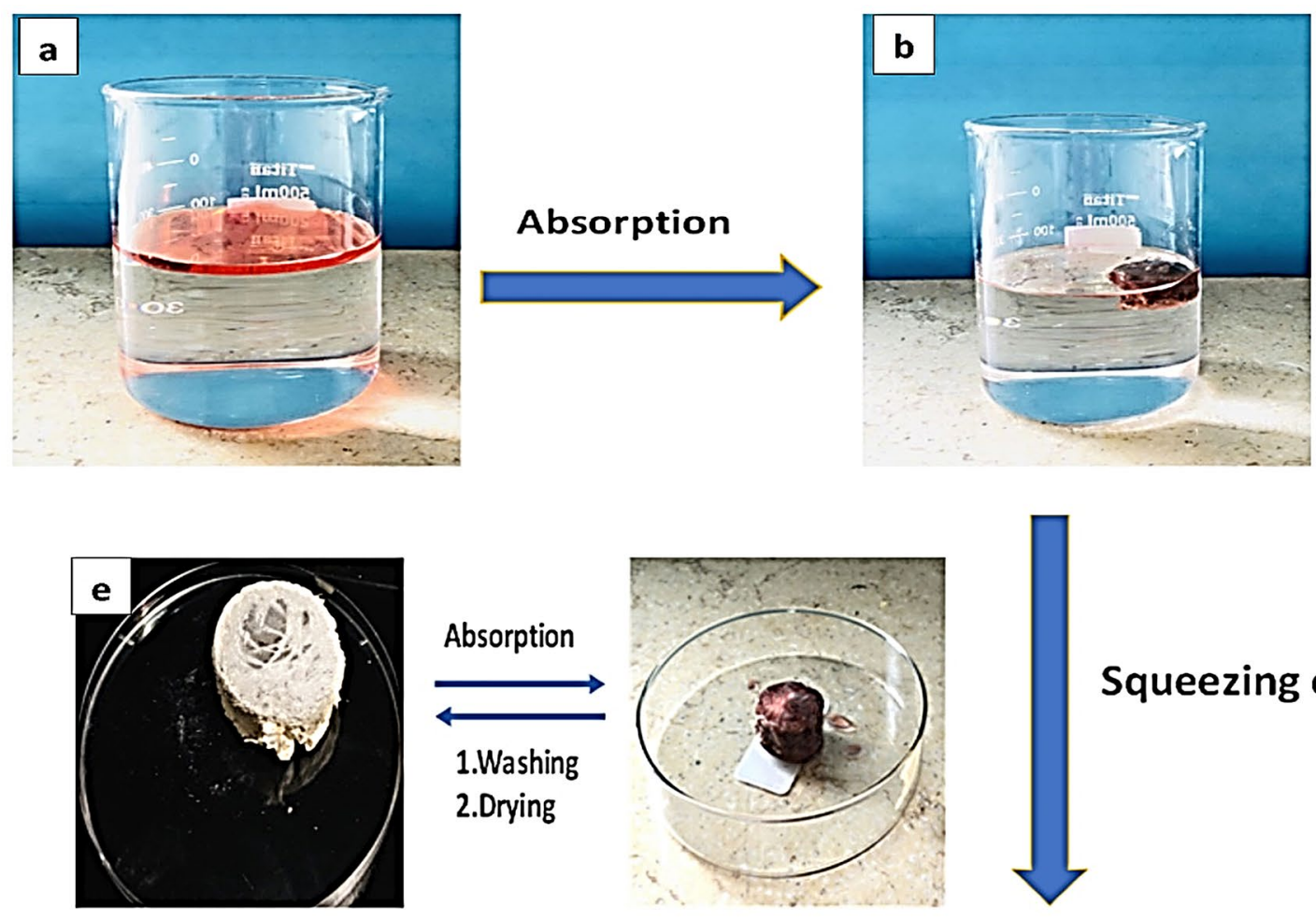

\section{Squeezing out}
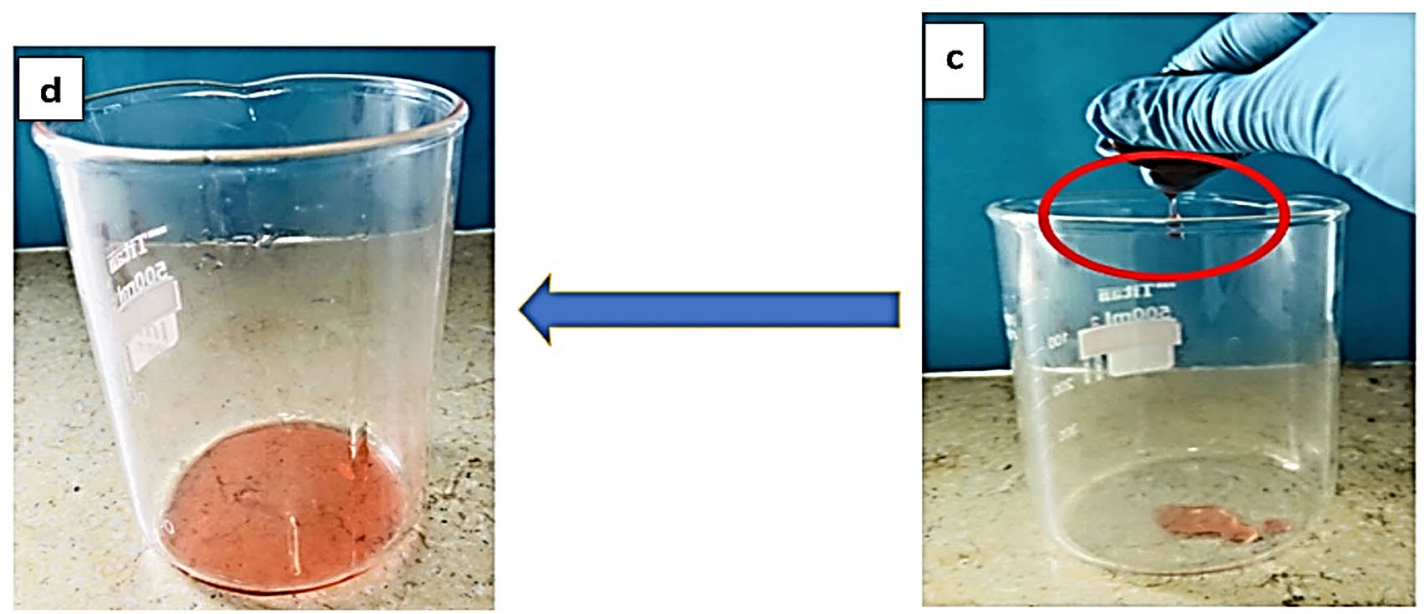

Fig. 6 The absorption and recycling process of soybean oil (oil dyed with red color) and the recovery of the PP 10L sponge by washing and drying in the air 


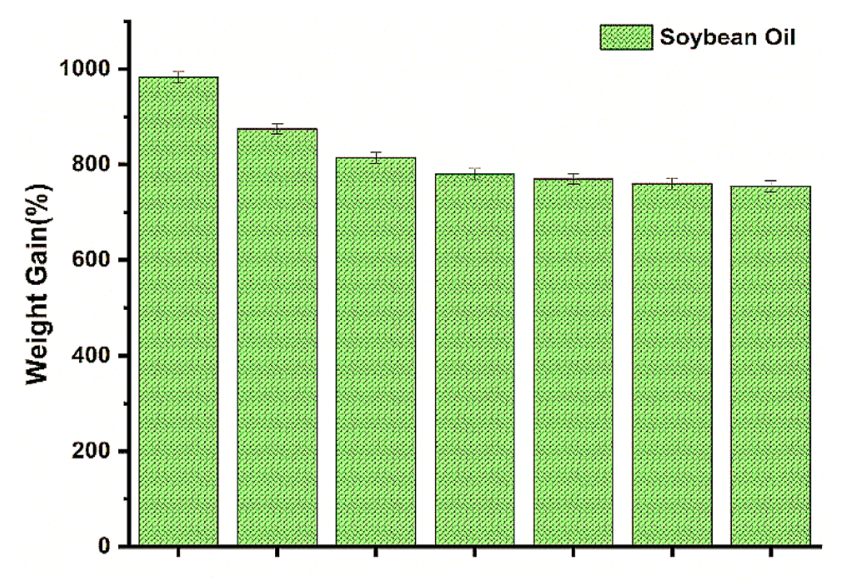

Cycle

Fig. 7 Absorption recyclability of PP 10L sponge toward soybean oil
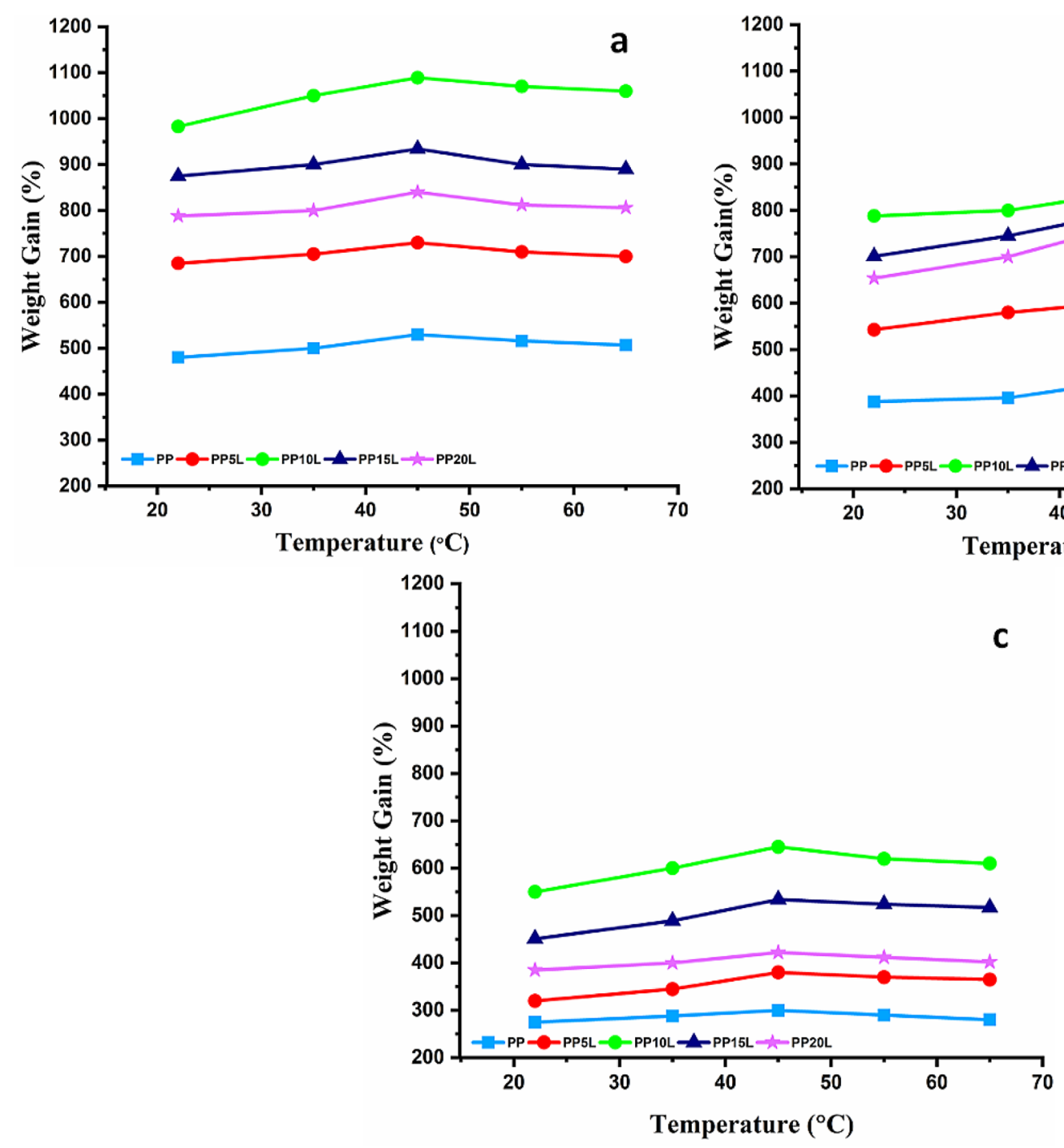

Fig. 8 Effect of temperature on oil absorption capacities of sponges a soybean oil, $\mathbf{b}$ engine oil, $\mathbf{c}$ lubricating oil surface of the water. Once contacted, the floating oil drop is quickly and selectively collected inside the blend material. The adsorbed oil gathered easily by hand squeezing, as shown in Fig. 6c. After repeating the process several times in water and oil, the oil was successfully separated. The sponge could be easily reused by washing and dried subsequently, as shown in Fig. 7, which considers valuable property for practical applications. As described above, the excellent absorption properties make sponge a recyclable oil sorbent for large-scale oil spill cleanup.

Temperature is a significant parameter in the oily wastewater research since the temperature differs due to areas and seasons [51]. In this study, the different temperature was taken in the range $22^{\circ} \mathrm{C}-35^{\circ} \mathrm{C}-45^{\circ} \mathrm{C}-55^{\circ} \mathrm{C}$ and $65^{\circ} \mathrm{C}$, respectively. The results of the experiments were presented, as shown in Fig. 8. It was shown the oil temperature effect on the weight gain of tested samples. It was observed that with an increase in oil temperature

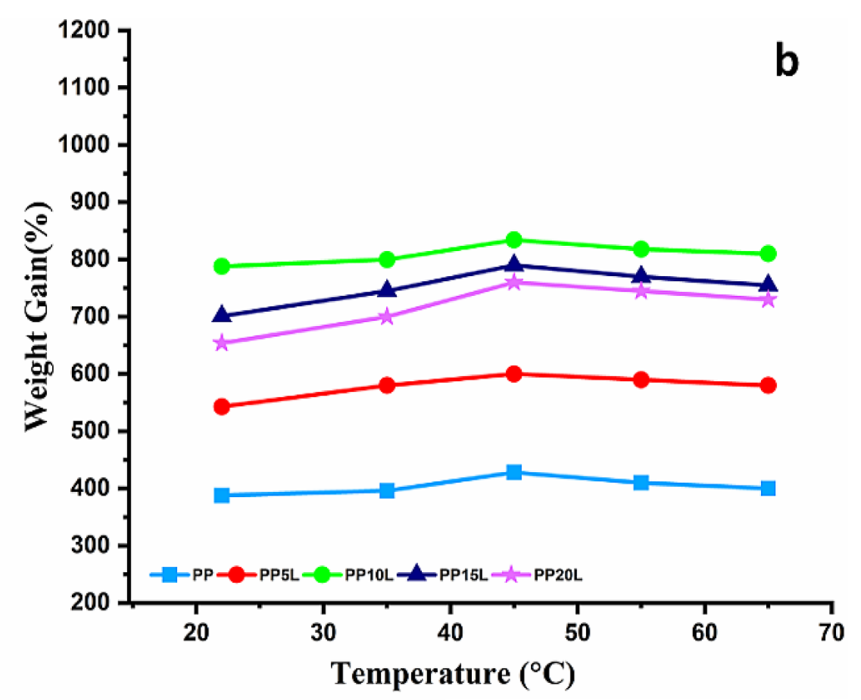


from $22^{\circ} \mathrm{C}$ until reaching temperature $45^{\circ} \mathrm{C}$, the oil uptake (weight gain) increased gradually. This indicates that oil viscosity is directly related to the temperature, which can be explained to the random motion of particles increase, which supports the opportunity of oil droplets to be attached on the sorbent surface and penetrate the pores. Furthermore, with increasing temperature beyond $45^{\circ} \mathrm{C}$, the weight gain of tested samples decreases, and oil will be released from the pores and surface of the sorbent due to oil [52].

\section{Conclusion}

In this study, novel porous sponges based on economically and commercially available PP and lignin were successfully fabricated using the low-cost, simple process, eco-friendly, so-called thermally induced phase separation method (TIPS). Phase separation using the TIPS method was achieved in a short time, which shows a great advantage for the cleanup of a sudden oil spill accident. SEM showed sponges with a three-dimensional interconnected porous structure and showed lignin mixed with polypropylene. FTIR analysis revealed the successful blend of polypropylene and lignin together. Sponges showed good adsorption ability to oils. The absorbed oils were easily recycled by squeezing the sorbents manually. Moreover, the weight gain (\%) of tested samples was found dependent on the temperature of the media. Considering oil absorbency of sponge, as well as the template-free and versatile fabrication method, this research presented a method for the design and fabrication of blend porous materials from polypropylene and lignin.

\section{Compliance with ethical standards}

Conflict of interest The authors declare that they have no conflict of interest.

\section{References}

1. Bayık GD, Altın A (2018) Conversion of an industrial waste to an oil sorbent by coupling with functional silanes. J Clean Prod 196:1052-1064

2. Bayık GD, Altın A (2017) Production of sorbent from paper industry solid waste for oil spill cleanup. Mar Pollut Bull 125(1):341-9

3. Wang G, Sun Q, Zhang Y, Fan J, Ma L (2010) Sorption and regeneration of magnetic exfoliated graphite as a new sorbent for oil pollution. Desalination 263:183-188

4. Bai X, Shen Y, Tian H, Yang Y, Feng H, Li J (2019) Facile fabrication of superhydrophobic wood slice for effective water-in-oil emulsion separation. Sep Purif Technol 210:402-408
5. Said AE, Ludwick AG, Aglan HA (2009) Usefulness of raw bagasse for oil absorption: a comparison of raw and acylated bagasse and their components. Biores Technol 100:2219-2222

6. Wahi R, Chuah LA, Choong TS, Ngaini Z, Nourouzi MM (2013) Oil removal from aqueous state by natural fibrous sorbent: an overview. Sep Purif Technol 113:51-63

7. Zhang H, Shen Y, Li M, Zhu G, Feng H, Li J (2019) Egg shell powders-coated membrane for surfactant-stabilized crude oilin-water emulsions efficient separation. ACS Sustain Chem Eng 7:10880-10887

8. Cui M, Mu P, Shen Y, Zhu G, Luo L, Li J (2020) Three-dimensional attapulgite with sandwich-like architecture used for multifunctional water remediation. Sep Purif Technol 235:116210

9. Davoodi SM, Taheran M, Brar SK, Galvez-Cloutier R, Martel R (2019) Hydrophobic dolomite sorbent for oil spill clean-ups: kinetic modeling and isotherm study. Fuel 251:57-72

10. Li M, Bian C, Yang G, Qiang X (2019) Facile fabrication of waterbased and non-fluorinated superhydrophobic sponge for efficient separation of immiscible oil/water mixture and water-inoil emulsion. Chem Eng J 368:350-358

11. Rotar OV, Iskrizhitskaya DV, Iskrizhitsky AA, Oreshina AA (2014) Cleanup of water surface from oil spills using natural sorbent materials. Proc Chem Chem Chem Eng 10:145-150

12. Liu F, Ma M, Zang D, Gao Z, Wang C (2014) Fabrication of superhydrophobic/superoleophilic cotton for application in the field of water/oil separation. Carbohydr Polym 103:480-487

13. Alassod A, Abedalwafa MA, Xu G (2020) Evaluation of polypropylene melt blown nonwoven as the interceptor for oil. Environ Technol 23:1-3

14. Guo M, Liang H, Luo Z, Chen Q, Wei W (2016) Study on meltblown processing, web structure of polypropylene nonwovens and its BTX adsorption. Fibers Polym 17:257-265

15. Renuka S, Rengasamy RS, Das D (2016) Studies on needlepunched natural and polypropylene fiber nonwovens as oil sorbents. J Ind Text 46:1121-1143

16. Xu M, Bian J, Han C, Dong L (2016) Hydrophobic modification of polypropylene/starch blend foams through tailoring cell diameter for oil-spill cleanup. RSC Adv 6:82088-82095

17. Blanco I, Cicala G, Latteri A, Saccullo G, El-Sabbagh AM, Ziegmann $G$ (2017) Thermal characterization of a series of ligninbased polypropylene blends. JTherm Anal Calorim 127:147-153

18. Dias OA, Sain M, Cesarino I, Leão AL (2019) Development of high bio-content polypropylene composites with different industrial lignins. Polym Adv Technol 30:70-78

19. Doherty WO, Mousavioun P, Fellows CM (2011) Value-adding to cellulosic ethanol: lignin polymers. Ind Crops Prod 33:259-276

20. Kurian JV (2005) A new polymer platform for the futureSorona from corn derived 1,3-propanediol. J Polym Environ 13:159-167

21. Nedelcu D, Ciofu C, Lohan NM (2013) Microindentation and differential scanning calorimetry of "liquid wood". Compos B Eng 55:11-15

22. Kalia S, Dufresne A, Cherian BM, Kaith BS, Avérous L, Njuguna J, Nassiopoulos E (2011) Cellulose-based bio-and nanocomposites: a review. Int J Polym Sci 2011:1-35

23. Laurichesse $S$, Avérous $L$ (2014) Chemical modification of lignins: towards biobased polymers. Prog Polym Sci 39:1266-1290

24. Faruk O, Sain M (2015) Lignin in polymer composites. William Andrew, Norwich

25. Liu H, Dai Z, Cao Q, Shi X, Wang X, Li H, Han Y, Li Y, Zhou J (2018) Lignin/polyacrylonitrile carbon fibers: the effect of fractionation and purification on properties of derived carbon fibers. ACS Sustain Chem Eng 6:8554-8562

26. Bozsódi B, Romhányi V, Pataki P, Kun D, Renner K, Pukánszky B (2016) Modification of interactions in polypropylene/lignosulfonate blends. Mater Des 103:32-39 
27. Sadeghifar H, Argyropoulos DS (2016) Macroscopic behavior of kraft lignin fractions: melt stability considerations for ligninpolyethylene blends. ACS Sustain Chem Eng 4:5160-5166

28. Maldhure AV, Ekhe JD (2017) Effect of modifications of lignin on thermal, structural, and mechanical properties of polypropylene/modified lignin blends. J Thermoplast Compos Mater 30:625-645

29. Hilburg SL, Elder AN, Chung H, Ferebee RL, Bockstaller MR, Washburn NR (2014) A universal route towards thermoplastic lignin composites with improved mechanical properties. Polymer 55:995-1003

30. Košíková B, Demianova V, Kačuráková M (1993) Sulfur-free lignins as composites of polypropylene films. J Appl Polym Sci 47:1065-1073

31. Chen F, Dai H, Dong X, Yang J, Zhong M (2011) Physical properties of lignin-based polypropylene blends. Polym Compos 32:1019-1025

32. Maldhure AV, Ekhe JD, Deenadayalan E (2012) Mechanical properties of polypropylene blended with esterified and alkylated lignin. J Appl Polym Sci 125:1701-1712

33. Wang G, Uyama H (2016) Facile synthesis of flexible macroporous polypropylene sponges for separation of oil and water. Sci Rep 6:1-6

34. Wang $G$, Xin $Y$, Uyama $H$ (2015) Facile fabrication of mesoporous poly (ethylene-co-vinyl alcohol)/chitosan blend monoliths. Carbohydr Polym 132:345-350

35. Okada K, Nandi M, Maruyama J, Oka T, Tsujimoto T, Kondoh K, Uyama $\mathrm{H}$ (2011) Fabrication of mesoporous polymer monolith: a template-free approach. Chem Commun 47:7422-7424

36. Wang G, Peng L, Yu B, Chen S, Ge Z, Uyama H (2018) Hierarchically porous sponge for oily water treatment: facile fabrication by combination of particulate templates and thermally induced phase separation method. J Ind Eng Chem 62:192-196

37. Kim JF, Kim JH, Lee YM, Drioli E (2016) Thermally induced phase separation and electrospinning methods for emerging membrane applications: a review. AIChE J 62:461-490

38. Peng M, Jia H, Jiang L, Zhou Y, Ma J (2019) Study on structure and property of PP/TPU melt-blown nonwovens. J Text Inst 110:468-475

39. Nguyen DD, Vu CM, Vu HT, Choi HJ (2019) Micron-size white bamboo fibril-based silane cellulose aerogel: fabrication and oil absorbent characteristics. Materials 12:1407

40. Cao S, Dong T, Xu G, Wang FM (2017) Oil spill cleanup by hydrophobic natural fibers. J Nat Fibers 14:727-735
41. Tsujimoto T, Hosoda N, Uyama H (2016) Fabrication of porous poly (3-hydroxybutyrate-co-3-hydroxyhexanoate) monoliths via thermally induced phase separation. Polymers 8:66

42. Zhang Y, Zhang Y, Cao Q, Wang C, Yang C, Li Y, Zhou J (2020) Novel porous oil-water separation material with super-hydrophobicity and super-oleophilicity prepared from beeswax, lignin, and cotton. Sci Total Environ 706:135807

43. Supanchaiyamat N, Jetsrisuparb K, Knijnenburg JT, Tsang DC, Hunt AJ (2019) Lignin materials for adsorption: current trend, perspectives and opportunities. Bioresour Technol 272:570-581

44. Jayaramulu K, Geyer F, Schneemann A, Kment Š, Otyepka M, Zboril R, Vollmer D, Fischer RA (2019) Hydrophobic metalorganic frameworks. Adv Mater 31:1900820

45. Yeo JS, Seong DW, Hwang SH (2015) Chemical surface modification of lignin particle and its application as filler in the polypropylene composites. J Ind Eng Chem 31:80-85

46. Gadioli R, Waldman WR, De Paoli MA (2016) Lignin as a green primary antioxidant for polypropylene. J Appl Polym Sci 133:45

47. Lu H, Cornell A, Alvarado F, Behm M, Leijonmarck S, Li J, Tomani $\mathrm{P}$, Lindbergh $\mathrm{G}$ (2016) Lignin as a binder material for eco-friendly Li-ion batteries. Materials 9:127

48. Sahoo S, Seydibeyoğlu MÖ, Mohanty AK, Misra M (2011) Characterization of industrial lignins for their utilization in future value added applications. Biomass Bioenergy 35:4230-7

49. Santos OS, da Silva MC, Silva VR, Mussel WN, Yoshida MI (2017) Polyurethane foam impregnated with lignin as a filler for the removal of crude oil from contaminated water. J Hazard Mater 324:406-413

50. Dong T, Cao S, Xu G (2016) Highly porous oil sorbent based on hollow fibers as the interceptor for oil on static and running water. J Hazard Mater 305:1-7

51. Abdelwahab O, Nasr SM, Thabet WM (2017) Palm fibers and modified palm fibers adsorbents for different oils. Alex Eng $J$ 56:749-755

52. Kudaybergenov KK, Ongarbayev EKMZ (2015) Oil sorption by heat-treated rice husks. J Pet Environ Biotechnol 6:1

Publisher's Note Springer Nature remains neutral with regard to jurisdictional claims in published maps and institutional affiliations. 